\title{
Some peculiarities of spin-wave propagation in magnonic waveguides
}

\author{
N. Grigoryeva ${ }^{1}$, D. Popov ${ }^{1}$ and B. Kalinikos ${ }^{1}$ \\ ${ }^{1}$ Saint-Petersburg Electrotechnical University, Department of Physical Electronics and Technology, 197376, Saint- \\ Petersburg, Russia
}

\begin{abstract}
A normal-mode theory for the dipole-exchange spin-wave spectrum in the finite-width ferromagnetic waveguide is presented. The theory takes into account a nonuniform character of the demagnetizing field in the waveguide cross section and, therefore, can be applied to any infinitely long, rectangular rod, even with square cross section. The inhomogeneity of static and dynamic dipole fields is taken into account using the same tensorial Green's function, obtained from Maxwell equations, this fact allows to simplify the spectrum calculation procedure. According to the elaborated theory the spin-wave spectrum in the finite-width ferromagnetic waveguide can be calculated with simultaneous account of the dipole-dipole and exchange interaction, surface anisotropy, arbitrary direction of the external bias magnetic field and for any possible width-thickness aspect ratio of the magnonic waveguide. It is shown that the previously used analytical methods of the accounting of the finite width of the magnetic waveguides give unsuitable results for nanometer-size waveguides.
\end{abstract}

\section{Introduction}

A demand of miniaturization in nanoelectronics leads to a size reduction of the ferromagnetic elements used in microwave devices. It is well known that the conditions of the wave propagation in confined waveguiding structures differ drastically from the infinite-film ones. In the magnetic finite-width waveguides a lot of new effects appear due to the strong confinement of propagating spin waves [1-4]. From these perspectives, commonly used approximate theoretical methods cannot meet the demands of the theoretical analysis of the obtained experimental data.

Here we report a normal-mode theory elaborated for the description of spin-wave propagation in a finite-width ferromagnetic waveguide. The dipole-exchange spectrum of spin waves is obtained analytically using the method of tensorial Green's function together with the spin-wave modes approach, presented in our previous works (see [5] and refs. therein). A finite width of the waveguiding structure is taken into account simultaneously with the arbitrary type of surface anisotropy on the edges of the waveguide. The problem of determination of the inhomogeneous internal static magnetic field in the confined structure is solved for an arbitrary direction of the external bias magnetic field and for any widththickness aspect ratio of the waveguide.

To illustrate the presented theoretical approach, the numerical calculations of the spin-wave spectrum were done for the most common case of tangentially magnetized waveguiding structure. It is demonstrated that the approximate analytical methods, which are widely used for the accounting of finite-size effects in ferromagnetic waveguides, give a large discrepancy for micron- and nanometer-size samples.

\section{Theoretical model}

Let us consider the magnonic waveguide of a thickness $L$ and width $w$. The length of the waveguide is supposed to be infinite in $\zeta$ direction (Fig. 1). The magnonic wavegiude is assumed to be magnetized to saturation by an arbitrary oriented bias external magnetic field.

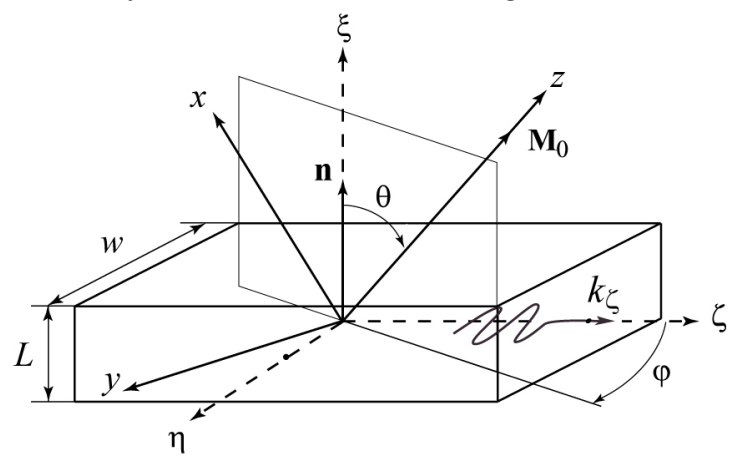

Fig. 1. Geometry of the problem.

For the convenience of further analysis two coordinate systems were introduced. One coordinate system, $\xi \eta \zeta$, is aligned to the sample geometry, with axes

This is an Open Access article distributed under the terms of the Creative Commons Attribution License 2.0, which permits unrestricted use, distribution, and reproduction in any medium, provided the original work is properly cited. 
$\xi$ and $\eta$ directed along the normal to the waveguide edges and axis $\zeta$ directed parallel to the spin-wave propagation direction. And another coordinate system, $x y z$, is associated with the equilibrium orientation of the saturation magnetization $\mathbf{M}_{0}$. The transition from one coordinate system to another can be done by means of orthogonal transformations of rotation through equilibrium angles $\varphi$ and $(\theta-\pi / 2)$.

The total effective magnetic field in the LandauLifshitz equation of motion for magnetization (see e.g. [5]) in the considered case have the following form:

$$
\mathbf{H}^{e f f}(\mathbf{r}, t)=\mathbf{H}_{0}+\mathbf{H}^{d}(\xi, \eta)+\mathbf{h}^{d}(\mathbf{r}, t)+\mathbf{h}^{e x}(\mathbf{r}, t),
$$

where $\mathbf{H}_{0}-$ is an external bias magnetic field, $\mathbf{H}^{d}(\xi, \eta)-$ is a static dipole (or demagnetizing) field, which have the variation in the waveguide cross section, $\mathbf{h}^{d}(\mathbf{r}, t)-$ is a dynamic dipole field, $\mathbf{h}^{e x}(\mathbf{r}, t)=\alpha \nabla^{2} \mathbf{m}(\mathbf{r}, t)-$ is a variable exchange field ( $\alpha$ is the exchange constant, $\mathbf{m}(\mathbf{r}, t)$ is a variable magnetization).

The solution of the considered problem is represented in the form of nonuniform plane waves:

$$
\mathbf{m}(\mathbf{r}, t)=\mathbf{m}\left(\xi, \eta ; k_{\zeta}\right) \exp \left[i\left(\omega t-k_{\zeta} \zeta\right)\right] \text {. }
$$

According to the method of tensorial Green's functions a Fourier amplitude of the dynamic dipole field $\mathbf{h}^{d}(\mathbf{r}, t)$ for the magnonic waveguide can be expressed through $\mathbf{m}\left(\xi, \eta ; k_{\zeta}\right)$ in the integral form [5]:

$$
\mathbf{h}^{d}\left(\xi, \eta ; k_{\zeta}\right)=\int_{-\frac{L}{2}-\frac{w}{2}}^{\frac{L}{2}} \int_{\frac{w}{2}}^{\frac{w}{\mathbf{G}}}\left(\xi, \xi^{\prime}, \eta, \eta^{\prime} ; k_{\zeta}\right) \mathbf{m}\left(\xi^{\prime}, \eta^{\prime} ; k_{\zeta}\right) d \eta^{\prime} d \xi^{\prime}
$$

where $\hat{\mathbf{G}}\left(\xi, \xi^{\prime}, \eta, \eta^{\prime} ; k_{\zeta}\right)$ - is a tensorial Green's function of Maxwell equations with corresponding boundary conditions. Its components in coordinate system, $\xi \eta \zeta$ have the following form:

$$
G_{l l^{\prime}}\left(\xi, \xi^{\prime}, \eta, \eta^{\prime} ; k_{\zeta}\right)=\int_{0}^{\infty} C_{l l^{\prime}}\left(\kappa, k_{\zeta}\right) F\left(\xi, \xi^{\prime}, \eta, \eta^{\prime} ; \kappa, k_{\zeta}\right) d \kappa,
$$

where the function $F\left(\xi, \xi^{\prime}, \eta, \eta^{\prime} ; \kappa, k_{\zeta}\right)$ is determined as:

$F=\frac{e^{-\sqrt{k_{\zeta}^{2}+\kappa^{2}}\left|\xi^{\prime}-\xi^{\prime}\right|}}{2 \pi \sqrt{k_{\zeta}^{2}+\kappa^{2}}}\left\{\begin{array}{l}\sin \left(\kappa\left(\eta-\eta^{\prime}\right)\right) \text { for } G_{l \eta} ; \\ \cos \left(\kappa\left(\eta-\eta^{\prime}\right)\right) \text { for others. }\end{array}\right.$

The coefficients $C_{l l^{\prime}}\left(\kappa, k_{\zeta}\right)$ can be found as follows:

$$
\begin{aligned}
& C_{\zeta \eta}\left(\kappa, k_{\zeta}\right)=-\frac{i k_{\zeta}}{\kappa} C_{\eta \eta}=-\frac{i \kappa}{k_{\zeta}} C_{\zeta \zeta}=i \kappa k_{\zeta}, \\
& C_{\xi \eta}\left(\kappa, k_{\zeta}\right)=-\frac{i \kappa}{k_{\zeta}} C_{\xi \zeta}=\kappa \sqrt{k_{\zeta}^{2}+\kappa^{2}} \operatorname{sign}\left(\xi-\xi^{\prime}\right) .
\end{aligned}
$$

The tenzorial Green's function is symmetric $G_{l l^{\prime}}=G_{l^{\prime} l}$ and the diagonal components obey the rule:

$$
\sum_{l} G_{l l}=2 \pi \delta\left(\xi-\xi^{\prime}\right) \delta\left(\eta-\eta^{\prime}\right)
$$

It should be noted, that contrary to the other approximate analytical approaches presented in Refs. [68], our exact tensorial Green's function have all nine nonzero components and describes the nonuniform dipole field in the arbitrary magnetized magnonic waveguide with any width-thickness aspect ratio (even equal unity).

In the framework of spin-wave modes approach the initial system of the equations consisting of linearized Landau-Lifshitz equation of motion, Maxwell equations in the magnetostatic limit and corresponding electrodynamic boundary conditions can be rewritten in the form of one matrix integro-differential equation for vector Fourier amplitude of variable magnetization:

$\hat{\mathbf{F}} \mathbf{m}\left(\xi, \eta ; k_{\zeta}\right)+\hat{\mathbf{T}} \mathbf{m}\left(\xi, \eta ; k_{\zeta}\right)=\mathbf{h}^{d}\left(\xi, \eta ; k_{\zeta}\right)$.

This equation is written in $x y z$-coordinate system, in which saturation magnetization is oriented along $z$-axis. Here $\hat{\mathbf{F}}-$ is a linear differential matrix operator and $\hat{\mathbf{T}}$ includes the frequency-dependent part:

$$
\begin{aligned}
& \hat{\mathbf{F}}=-\alpha\left(\nabla_{\xi}^{2}+\nabla_{\eta}^{2}-k_{\zeta}^{2}\right)\left(\begin{array}{ll}
1 & 0 \\
0 & 1
\end{array}\right), \\
& \hat{\mathbf{T}}=\frac{1}{\gamma M_{0 z}}\left\{\left(H_{0 z}+H_{z}^{d}(\xi, \eta)\right)\left(\begin{array}{ll}
1 & 0 \\
0 & 1
\end{array}\right)+i \omega\left(\begin{array}{cc}
0 & -1 \\
1 & 0
\end{array}\right)\right\},
\end{aligned}
$$

where $H_{z}^{d}(\xi, \eta)$ and $H_{0 z}$ are the $z$-projections of the external bias magnetic field and the demagnetizing field, respectively. $\gamma=|g| \mu_{0},|g|$ is the modulus of the gyromagnetic ratio for electron spin and $\mu_{0}$ is the permeability of vacuum.

To derive the expression for the dipole-exchange spin-wave spectrum of a magnonic waveguide one should integrate the equation (6) along with the exchange boundary conditions, which depend of surface anisotropy. For further calculations we use Rado-Weertman exchange boundary conditions at all edges of the waveguide [9].

In accordance with the spin-wave modes approach, vector function $\mathbf{m}\left(\xi, \eta ; k_{\zeta}\right)$ in the coordinate system $x y z$, related with the equilibrium orientation of saturation magnetization, have only two components and can be expanded in the infinite series of a complete set of the orthogonal vector functions, so called spin-wave modes:

$\mathbf{m}\left(\xi, \eta ; k_{\zeta}\right)=M_{0 z} \sum_{n}^{\infty} \sum_{q}^{\infty} \sum_{p} m_{n q}^{p}\left(k_{\zeta}\right) \mathbf{S}_{n q}^{p}(\xi, \eta)$.

where spin-wave modes

$$
\mathbf{S}_{n q}^{x}=\left(\begin{array}{c}
\Phi_{n q}^{x}(\xi, \eta) \\
0
\end{array}\right), \mathbf{S}_{n q}^{y}=\left(\begin{array}{c}
0 \\
\Phi_{n q}^{y}(\xi, \eta)
\end{array}\right)
$$

are the eigenfunctions of the matrix-differential operator $\hat{\mathbf{F}}$, which satisfy mixed exchange boundary conditions.

In the linear case functions $\Phi_{n q}^{x, y}(\xi, \eta)$ became factorisable and can be expressed as a product of two functions: 


$$
\begin{aligned}
& \Phi_{n q}(\xi, \eta)=\Phi_{n}(\xi) \Phi_{q}(\eta)= \\
& =A_{n}\left[\cos \left(k_{n}(\xi+L / 2)\right)+\frac{d_{1}}{k_{n}} \sin \left(k_{n}(\xi+L / 2)\right)\right] \times \\
& \times B_{q}\left[\cos \left(\kappa_{q}(\eta+w / 2)\right)+\frac{d_{2}}{\kappa_{q}} \sin \left(\kappa_{q}(\eta+w / 2)\right)\right] .
\end{aligned}
$$

Here we omit upper indices $x, y$, because we assume that both components of variable magnetization are pinned uniformly $\Phi_{n q}^{x}(\xi, \eta)=\Phi_{n q}^{y}(\xi, \eta)$. The constants $A_{n}$ and $B_{q}$ are obtained from the normalization condition for spin-wave modes, and transverse wave numbers $k_{n}$ and $\kappa_{q}$ can be found as a solutions of transcendental equation, obtained from corresponding exchange boundary conditions. For more details see [5].

Substituting the expression for variable magnetization (9) to the linearized equation of motion (6) and using the normalization condition we arrive to the following infinite system of the linear algebraic equations:

$$
\widehat{\mathbf{D}}_{n q, n q} \mathbf{m}_{n q}+\sum_{n^{\prime} \neq n, q^{\prime} \neq q} \widehat{\mathbf{R}}_{n q, n^{\prime} q^{\prime}} \mathbf{m}_{n^{\prime} q^{\prime}}=0 .
$$

Linear matrix operators in (12) have the following form:

$$
\begin{aligned}
& \hat{\mathbf{D}}_{n q, n q}=\left[\begin{array}{cc}
F_{n q, n q}-\Gamma_{n q, n q}^{x x} & -\Gamma_{n q, n q}^{x y}-i \omega \\
-\Gamma_{n q, n q}^{y x}+i \omega & F_{n q, n q}-\Gamma_{n q, n q}^{y y}
\end{array}\right], \\
& \hat{\mathbf{R}}_{n q, n^{\prime} q^{\prime}}=\left[\begin{array}{cc}
F_{n q, n^{\prime} q^{\prime}}-\Gamma_{n q, n^{\prime} q^{\prime}}^{x x} & -\Gamma_{n q, n^{\prime} q^{\prime}}^{x y} \\
-\Gamma_{n q, n^{\prime} q^{\prime}}^{y x} & F_{n q, n^{\prime} q^{\prime}}-\Gamma_{n q, n^{\prime} q^{\prime}}^{y y}
\end{array}\right],
\end{aligned}
$$

and matrix elements can be derived as follows:

$$
\begin{aligned}
& F_{n q, n^{\prime} q^{\prime}}=\gamma H_{0 z} \delta_{n n^{\prime}} \delta_{q q^{\prime}}+ \\
& +\frac{\gamma}{w L} \int_{-\frac{L}{2}-\frac{w}{2}}^{\frac{L}{2}} \int_{n q}^{\frac{w}{2}} \Phi_{n}(\xi, \eta) H_{z}^{d}(\xi, \eta) \Phi_{n^{\prime} q^{\prime}}(\xi, \eta) d \xi d \eta, \\
& \Gamma_{n q, n^{\prime} q^{\prime}}^{\alpha \beta}=\frac{\gamma}{w L} \int_{-\frac{L}{2}-\frac{w}{2}-\frac{L}{2}-\frac{w}{2}}^{\frac{L}{2}} \int_{n q}^{\frac{L}{2}} \int^{\frac{w}{2}} \Phi_{n q}(\xi, \eta) M_{0 z} \times \\
& \times G_{n q, n^{\prime} q^{\prime}}^{\alpha \beta}\left(\xi, \xi^{\prime} ; \eta, \eta^{\prime} ; k_{\zeta}\right) \Phi_{n^{\prime} q^{\prime}}\left(\xi^{\prime}, \eta^{\prime}\right) d \eta d \xi d \eta^{\prime} d \xi^{\prime} .
\end{aligned}
$$

The analytical expressions for matrix elements (15), (16) can be derived analytically for each special case.

The infinite system (12) gives the exact description of the linear spin-wave processes in the magnonic waveguide and enables one to obtain the spectrum of normal modes in such structure and the transverse distribution of variable magnetization in the waveguide cross section.

\section{Results and discussion}

The condition of vanishing of the determinant of the infinite system (12) yields an exact dispersion relation for spin waves, propagating in the finite-width magnonic waveguide. Thus, the problem of determination of the dispersion characteristics of spin waves is reduced to the calculation of eigenvalues of the block matrix of coefficients of the linear algebraic system (12).

The obtained spin-wave spectrum takes into account both dipole-dipole and exchange interaction, since in micro- and nano-sized magnonic waveguides the normal modes have a mixed dipole-exchange nature when the lateral sizes or the characteristic localization lengths are comparable to the exchange length of the magnetic material [8]. In addition, the nonuniform character of the internal static magnetic field is taken into account with an arbitrary profile of the distribution of static magnetization in the waveguide cross section. Moreover, the mixed exchange boundary conditions are applied at all surfaces of the waveguide, to obtain the solutions for partially pinned surface spins on lateral edges.

\subsection{Static problem}

One of the main problems in the theoretical description of the spin-wave spectra in the finite-width magnonic waveguide is the calculation of spatially nonuniform static demagnetizing field inside the waveguide. The first attempt to obtain the explicit expression for the demagnetizing field in non-ellipsoidal ferromagnetic element has been undertaken by Joseph and Schlomann [10]. In the case of the uniformly magnetized infinitely long magnonic waveguide the expression for the tensor of demagnetizing factors as in Ref. [10] can be obtained from Eq. (3) by the evaluation of integration over ' $\xi$ ' and $\eta^{\prime}$ with following substitution: $\mathbf{m}\left(\xi^{\prime}, \eta^{\prime} ; k_{\zeta}\right) \rightarrow M_{0}$, $\mathcal{G}\left(\xi, \xi^{\prime}, \eta, \eta^{\prime} ; k_{\zeta}\right) \rightarrow \mathcal{G}\left(\xi, \xi^{\prime}, \eta, \eta^{\prime} ; k_{\zeta}=0\right)$.

It is worth nothing that the expression (3), obtained in the framework of tensorial Green's functions approach, stay valid for any width-thickness aspect ratio. Moreover, the static internal magnetic field can be correctly calculated for any spatial distribution of static magnetization (not only the constant).

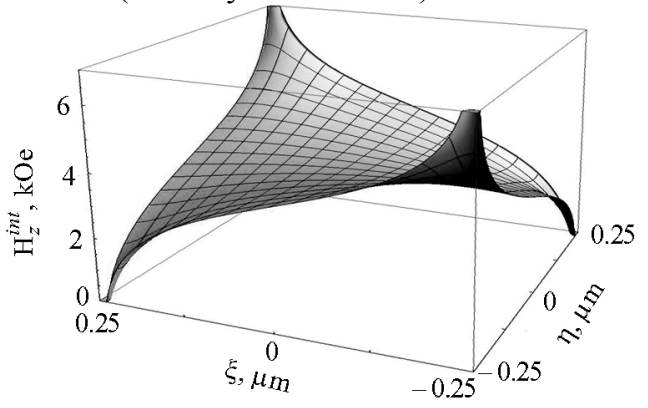

Fig. 2. Cross-sectional distribution of the z-component of the static internal magnetic field.

In Fig. 2 the calculated cross-sectional distribution of the $z$-component of the static internal magnetic field is presented for the infinitely long Permalloy waveguide $\left(\mathbf{M}_{0}=4 \pi \cdot 758 \mathrm{G}\right)$ with square cross section 
$w \times L=0.5 \times 0.5 \mu \mathrm{m}$. The internal field was calculated for the case of the uniformly magnetized ferromagnetic waveguide and for the external bias magnetic field $H_{0}=7 \mathrm{kOe}$ oriented in the direction $\theta=\pi / 4, \varphi=\pi / 4$.

From Fig. 2 it is well seen that any averaging by thickness, used by authors in Refs. [6-8] is impossible in the case of an arbitrary magnetized magnonic waveguide. In this case the full account of two-dimensional distribution of the internal magnetic field have to be done in order to describe correctly the conditions of spinwaves propagation.

\subsection{Dynamic problem}

To obtain numerically the spectrum of spin-waves the infinite system of the equations (12) can be reduced to the finite one by taking into account the limiting number of spin-wave modes. Such a truncation is justified by physical conditions of excitation and propagation of different spin-wave modes in each particular case. It is well known, that the efficiency of excitation for different spin-wave modes is different and also depends on the excitation source. The higher spin-wave modes usually have less excitation efficiency and low group velocity, thus cannot influence much on the final result. On the other hand, the strong dipole-dipole interaction of the main spin-wave mode with higher modes may change drastically the initial spectrum of non-interacting modes. In the framework of spin-wave modes approach the satisfying results for the spectrum of interacting normal modes can be obtained while last five spin-wave modes are discard, i.e. $n \times n$ matrix results in $n-5$ correct normal modes.

As an example of the successful implementation of the elaborated theory, the dipole-exchange spin-wave spectrum for the magnonic waveguide, investigated in [11], is presented in Fig. 3. The authors [11] use a wellknown and commonly used method of the theoretical descriprion of the spin-wave spectrum of the magnonic waveguide by means of the modified expressions, obtained for the infinite film. The discrepancy is obvious.

Without introducing effective parameters (effective width and effective magnetic field) the commonly used theory cannot describe even the FMR frequency of propagating spin-waves. Moreover, after the introduction of the effective parameters, which became now unknown fitting parameters, the commonly used theory still doesn't give an appropriate result. It is well seen that in the longwavelength region there is strong dipole-dipole intermode interaction, which the common theory doesn't take into acount. And in the short-wavelength region the group velocity of higher spin-wave modes differ from those calculated by the exact relations.

In Fig. 3 thin solid lines indicate the results of the exact theory for real parameters of the waveguide from Ref. [11] $\left(w=4.1 \mu \mathrm{m}, L=40 \mathrm{~nm}, \quad 4 \pi M_{0}=9525 \mathrm{G}\right.$, $\left.H_{0}=300 \mathrm{Oe}\right)$. The spin-wave spectrum calculated according to approximate method with the effective parameters $w_{\text {eff }}=3 \mu \mathrm{m}, H_{\text {eff }}=240 O e$ is shown by dashed lines. Thick solid line corresponds to the first thickness mode of the infinite film with free surface spins. The insert shows the geometry of the structure.

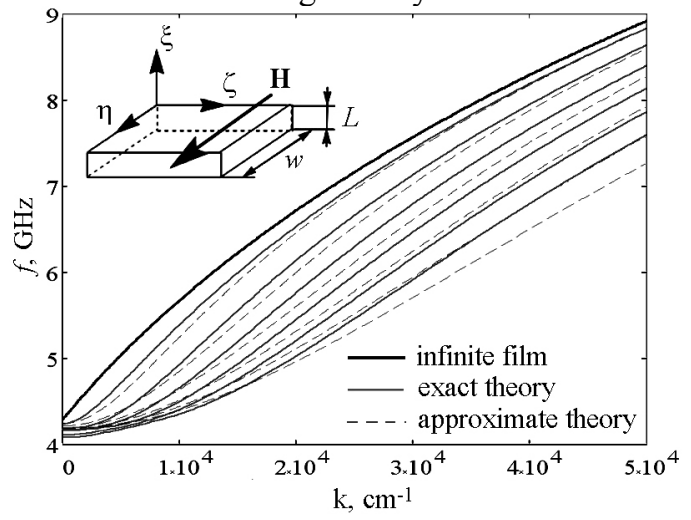

Fig. 3. Spin-wave spectrum calculated by means of different methods with the parameters of the magnonic waveguide taken from Ref. [11].

\section{Conclusion}

The exact dipole-exchange theory for spin waves, propagated in finite-width ferromagnetic waveguide is presented. The numeric results for spatial distribution of the inhomogeneous internal magnetic field and the exact spin-wave spectrum are obtained. The discrepancy between commonly used approximate theory and presented exact theory is demonstrated and discussed.

\section{References}

1. V.E. Demidov, S.O. Demokritov, K. Rott, P. Krzysteczko, G. Reiss, J. Phys. D: Appl. Phys. 41, 164012 (2008)

2. T. Bracher, P. Pirro, B. Obry, B. Leven, A.A. Serga, B. Hillebrands, Appl. Phys. Lett. 99, 162501 (2011)

3. F. Ciubotaru, A.V. Chumak, N.Yu. Grigoryeva, A.A. Serga, B. Hillebrands, J. Phys. D: Appl. Phys. 45, 255002 (2012)

4. M. Krawczyk, JMMM 322, 562 (2010)

5. N.Yu. Grigorieva, B.A. Kalinikos, In Magnonics: From Fundamentals to Applications, ed. by S.O. Demokritov, A.N. Slavin (Springer, NY, 2012), ISBN 3642302467

6. C. Mathieu, J. Jorzick, A. Frank, S.O. Demokritov, A.N. Slavin, B. Hillebrands, B. Bartenlian, C. Chappert, D. Decanini, F. Rousseaux, E. Cambril, Phys.Rev. Lett. 81, 3968 (1998)

7. M.P. Kostylev, G. Gubbiotti, J.-G. Hu, G. Carlotti, T. Ono, R. L. Stamps, Phys. Rev. B 76, 054422 (2007)

8. K.Y. Guslienko, A.N. Slavin, JMMM 323, 2418 (2011)

9. G.T. Rado, J.R. Weertman, J. Phys. Chem. Solids 11, 315 (1959)

10. R. I. Joseph, E. Schlomann, J. Appl. Phys. 36, 1579 (1965)

11. P. Pirro, T. Brachery, K. Vogt, B. Obry, H. Schultheiss, B. Leven, B. Hillebrands, Phys. Status Solidi B, 1 (2011) 